www.jmscr.igmpublication.org

Impact Factor 5.244

Index Copernicus Value: 5.88

ISSN (e)-2347-176x ISSN (p) 2455-0450

crossref DOI: _http://dx.doi.org/10.18535/jmscr/v4i4.52

Journal Of Medical Science And Clinical Research

\title{
Deliberate Self harm in Alcohol Dependent Outpatients in Tertiary Care Centre
}

\author{
Padmini Cherukunnath $^{1}$, Arun M Palayat ${ }^{2}$, Binoo Divakaran ${ }^{3}$, Mohanchandran V V ${ }^{4}$ \\ ${ }^{1}$ Assistant Prof in Psychiatry, Academy of Medical Sciences, Pariyaram Medical College, Kannur, Kerala \\ Email:ajimini2@gmail.com \\ ${ }^{2}$ Associate Prof in Psychiatry, Academy of Medical Sciences, Pariyaram Medical College, Kannur, Kerala \\ Email: arunpalayat@gmail.com
}

${ }^{3}$ Assistant Prof in Biostatistics, Academy of Medical Sciences, Pariyaram Medical College, Kannur, Kerala Email: binoovimal@gmail.com

${ }^{4}$ Professor of Psychiatry, Yenopoya Medical College, Mangalore, Karnataka

Email: mohanchandran660@yahoo.com

Dept of Psychiatry, Academy of Medical Sciences, Pariyaram Medical College, Kannur, Kerala, India

Corresponding Author

\section{Dr Padmini Cherukunnath,}

Assistant Professor in Psychiatry, Academy of Medical sciences, Pariyaram Medical college (PO),

Kannur, Pin Code: 670503. Kerala State, India.

Email: ajimini2@gmail.com.9447248774 / 9744167466

\begin{abstract}
Deliberate self harm (DSH) is a behavioral problem with significant clinical and public health importance. It is reported across several psychiatric illnesses like personality disorders, alcohol dependence syndrome $(A D S)$ and affective disorders. A hospital based cross-sectional study was carried out to find the incidence of DSH in outpatients with alcohol dependence syndrome, to learn about the methods used for DSH and to describe the association of DSH with psychiatric co-morbidity in the study population. Data was collected from 100 consecutive outpatients with ADS using structured socio-demographic Performa and semistructured pre-validated DSH questionnaire. ADS and psychiatric co-morbidities were diagnosed by International Classification of Diseases Diagnostic criteria for research- Tenth Revision. (ICD-10 DCR) Incidence of DSH in them in 1 year was 25\%. The commonest method of deliberate self-harm was consuming poison (76\%). 84\% had done DSH under intoxication.92\% of them had stressful situation immediately before DSH. $48 \%$ had attempted DSH impulsively. $40 \%$ had past history of psychiatric disorder. 56\% had past history of DSH. 56\% had psychiatric co morbidities, of which Depressive disorders (39\%) were the most common. 39\% of patients with psychiatric co morbidity had past history of DSH. 16\% with co morbid depressive disorder had attempted DSH in past 1 year. All patients presenting after deliberate self-harm need to be carefully screened for alcohol disorders and for co morbid psychiatric diagnoses. Abstaining from alcohol may be considered as a key to the resolution of deliberate self harm.

Keywords: Deliberate Self-Harm, Alcohol Dependence Syndrome, Psychiatric Co Morbidity.
\end{abstract}




\section{INTRODUCTION}

"Deliberate Self-Harm," (DSH) is defined as "self-poisoning or injury, irrespective of the purpose of the act. ${ }^{[1]}$ As in many countries, DSH in India is an unrecognized, hidden, and a silent epidemic. Incidence of DSH is increasing and is being reported in much younger age group, both in clinical and general population. ${ }^{[2]}$ Individuals who engage in DSH report using problematic coping mechanisms, one of which is alcohol consumption. ${ }^{[1]}$ Research on alcohol involvement and deliberate self-harm is conflicting. ${ }^{[3]}$ Alcohol dependence as well as co morbid psychopathology and negative life events, act as the distal risk factors for suicidal behavior. Proximal risk factors determine the timing of suicidal behavior by translating potential of distal risk factors into action. ${ }^{[4]}$ Acute effects of alcohol intoxication act as important proximal risk factor for suicidal behavior for patients with and without alcohol dependence. Alcohol consumption prior to suicide attempt is more important risk factor than the habitual alcohol consumption pattern. In addition to increasing likelihood of an attempt, alcohol may add considerably to danger of overdoses. [5] Mechanisms responsible for alcohol's ability to increase proximal risk for suicidal behavior include alcohol's ability to increase psychological distress, increase aggressiveness, propel suicidal ideation into action through suicide-specific alcohol expectancies and constrict cognition, which impairs generation and implementation of alternative coping strategies. [4] Alcohol dependence, depression and antisocial personality disorder form the formidable triad towards selfdestruction. [5] Methods employed for suicide attempt / self-harm in India are strikingly different from those reported in Western data. ${ }^{[6]}$

Alcohol dependent patients with DSH present a considerable challenge for clinical services. ${ }^{[4]} \mathrm{No}$ published study conducted in this region examined both issues of the presence of psychiatric co- morbidity and deliberate self harm in patients with alcohol dependence.

\section{AIMS \& OBJECTIVES}

1. To study the incidence and methods of deliberate self-harm in outpatients with alcohol dependence syndrome in a tertiary care centre.

2. To analyze the relationship between psychiatric co-morbidity and DSH in this population.

\section{MATERIALS \& METHODS}

This is a cross sectional hospital based study. Participants were recruited by purposive sampling based on the inclusion and exclusion criteria for a study period of 6 months. Cases for this study included 100 consecutive participants, aged 18-65 years, who met the ICD 10 DCR diagnostic criteria for alcohol dependence syndrome, who were willing to participate in the study and who had given written informed consent. Patients with co morbid organic mental disorders, severe physical problems and those who were unable to give written consent were excluded from the study. All patients were under the management of a consultant psychiatrist. Participants were interviewed with Socio- demographic Performa and DSH Questionnaire specially prepared by the researcher for this purpose. Past history and family history of ADS, or psychiatric disorders, or suicide and deliberate self harm, methods and details of attempts were recorded. Face-to-face interview rather than self-administered format was used to enhance cultural, language and literacy sensitivity. The draft schedule was given to 5 experts in the field for face validation. Based on their suggestions and comments, final schedule was prepared. Detailed history and mental state examination was done for all patients to diagnose psychiatric co-morbidities as per ICD-10 DCR.

Data collected was entered in and later analyzed using the computer program, Statistical Packages for Social Sciences (SPSS) Version 13. Descriptive statistical tools like frequency, percentage, mean and standard deviation were used. Categorical variables were analyzed using Chi- square or Fisher's exact test and continuous 
variables were analyzed using student's t test. A p value $<0.05$ was considered significant.

\section{RESULTS}

The study group comprised of 100 patients whose age ranged from 22 to 60 years, with a mean of 41.38 years and a standard deviation of 9.45 years. Majority of the patients were in the age group of 41-50 yrs (40\%). Hindus $(74 \%)$ dominated the sample. (Figure1). Majority (98\%) of the sample were males. Only 2 females were there in the study group who were Hindus in the age group of 41-50 years. Majority of the patients had primary education $(72 \%)$, were employed $(66 \%)$, married $(65 \%)$, and hailed from rural areas $(74 \%)$ and joint families $(60 \%)$. (Table 1 )

Out of 100 patients in the sample, 25 patients had attempted DSH in the past 1 year (25\%).Table: 2 shows characteristics of patients with history of deliberate self harm (DSH) in last one year. The commonest method of deliberate self-harm were consuming poison (76\%). 92\% had history of stressful situation immediately before the attempt. $84 \%$ were under intoxication when they attempted DSH. $40 \%$ of the sample had past history of a psychiatric disorder and 56\% had past history of deliberate self harm. $48 \%$ reported attempt as an impulsive act.

$79 \%$ patients had co morbid nicotine dependence. $56 \%$ had psychiatric co morbidities. Depressive disorders (39\%) were the most common psychiatric co morbidity encountered. (Figure 2) Patients with psychiatric co morbidity were more likely to have DSH (39\%). (Figure 3) Table: 3 shows that higher number of patients with co morbid depressive disorders or adjustment disorders had attempted DSH in past 1 year when compared with patients with other co morbid psychiatric diagnosis and this relation was statistically significant.

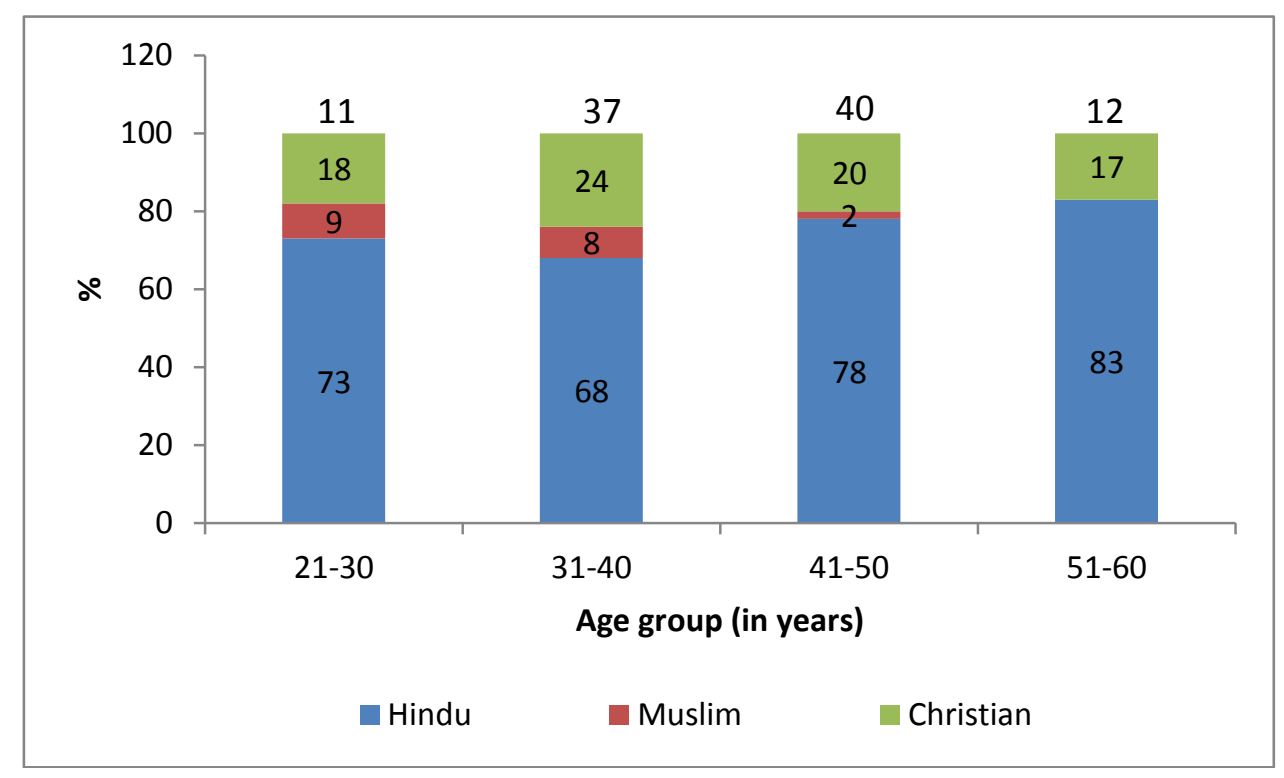

Figure: 1 Age group and religion wise distribution 


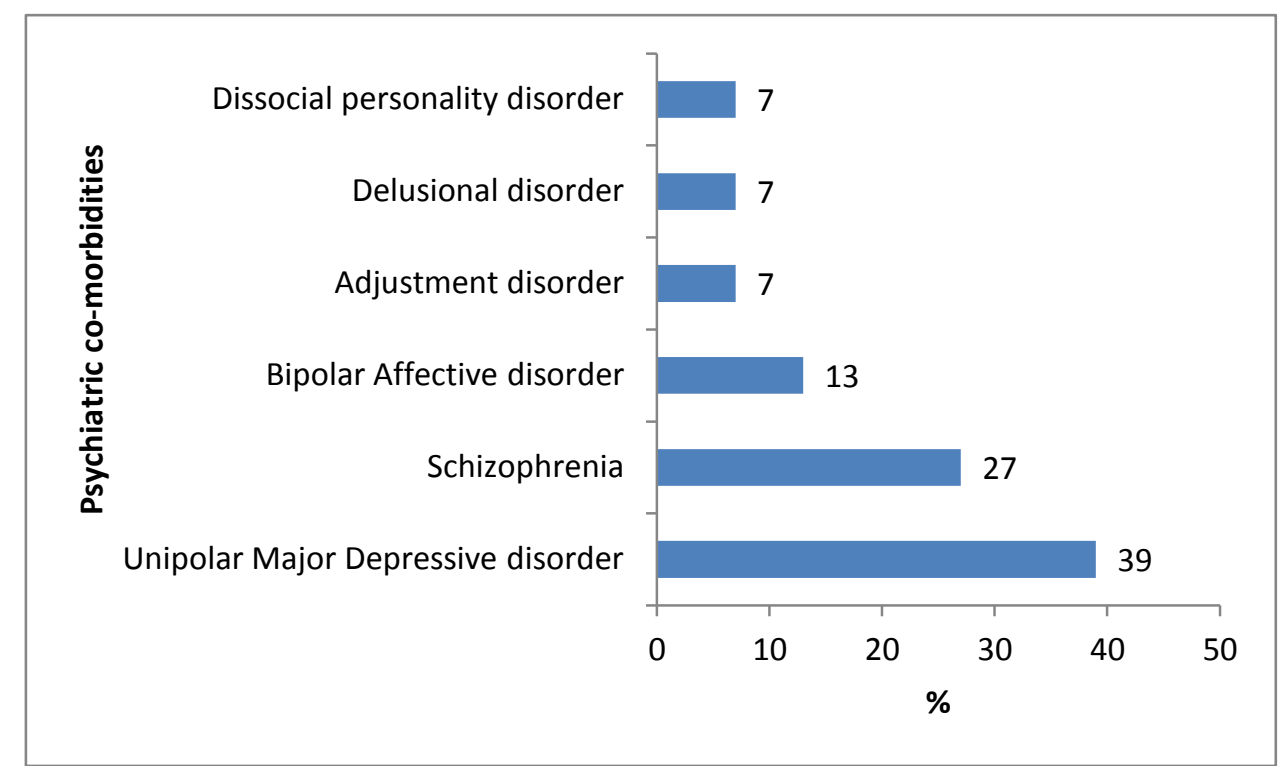

Figure: 2. Psychiatric co morbidity in the sample

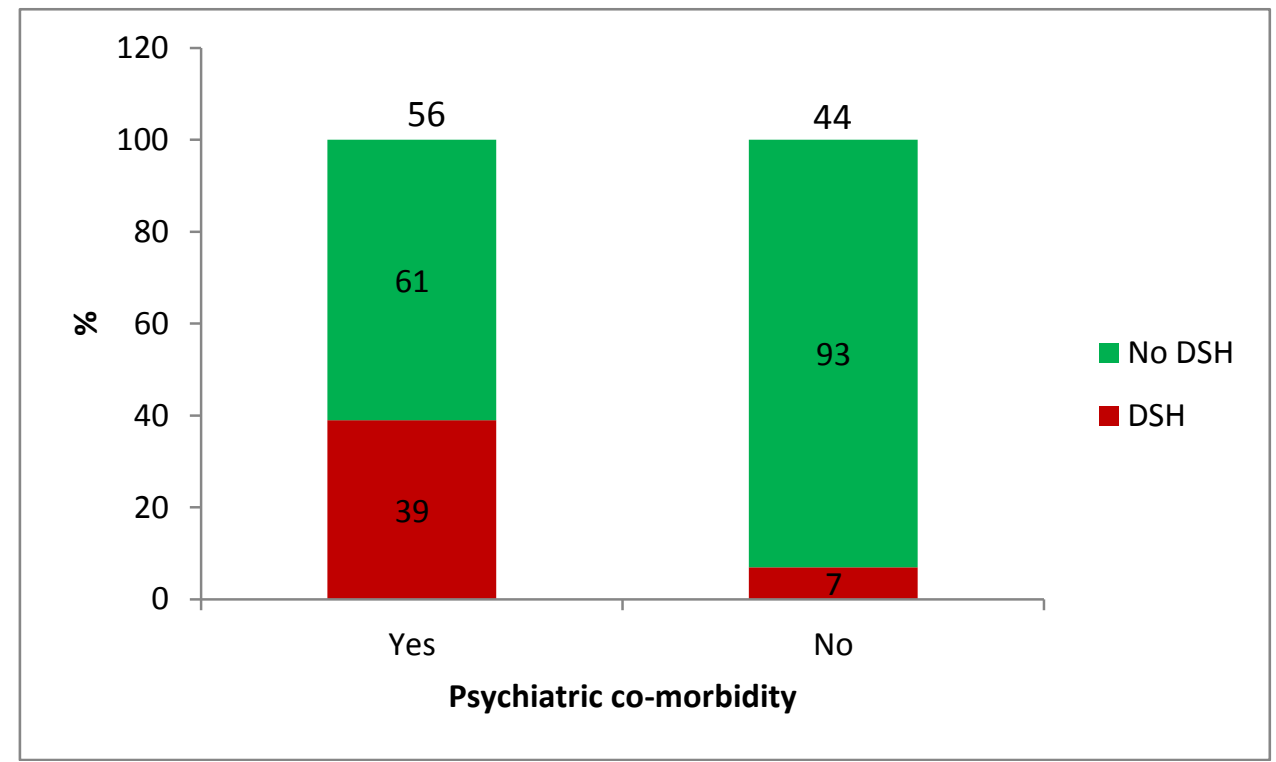

Figure: 3. Deliberate self harm (DSH) and psychiatric co morbidity 
Table: 1. Socio- demographic data

\begin{tabular}{|llll|}
\hline Variables & & N & $\%$ \\
\hline Sex & Male & 98 & 98 \\
& Female & 2 & 2 \\
\hline Marital Status & Single & 22 & 22 \\
& Married & 65 & 65 \\
& Separated & 13 & 13 \\
\hline Educational Status & Primary & 72 & 72 \\
& Above primary & 28 & 28 \\
\hline Domicile & Urban & 26 & 26 \\
& Rural & 74 & 74 \\
\hline Occupation & Unemployed & 34 & 34 \\
& Employed & 66 & 66 \\
\hline Family type & Nuclear & 40 & 40 \\
& Joint & 60 & 60 \\
\hline
\end{tabular}

Table: 2. Characteristics of patients with history of DSH

\begin{tabular}{|c|c|c|}
\hline Characteristics & $\mathrm{N}=25$ & $\%$ \\
\hline Intoxicated at the time of DSH & 21 & 84 \\
\hline Impulsive act & 12 & 48 \\
\hline Stressor & 23 & 92 \\
\hline Past history of DSH & 14 & 56 \\
\hline Family history of DSH & 18 & 72 \\
\hline Past history of psychiatric illness & 10 & 40 \\
\hline Organo phosphorus & 17 & 68 \\
\hline Organochlor compounds & 2 & 8 \\
\hline Self inflicted cuts & 3 & 12 \\
\hline Acids & 3 & 12 \\
\hline
\end{tabular}

Table: 3. Psychiatric co-morbidity and DSH

\begin{tabular}{|c|c|c|c|c|c|}
\hline \multirow{3}{*}{$\begin{array}{l}\text { Psychiatric } \\
\text { morbidity }\end{array}$} & & \multicolumn{3}{|c|}{ Deliberate self harm } & \multirow[t]{3}{*}{ Significance } \\
\hline & & \multicolumn{2}{|c|}{ Yes } & \multirow{2}{*}{$\begin{array}{l}\text { No } \\
\%\end{array}$} & \\
\hline & $\mathrm{n}$ & $\%$ & $\mathrm{n}$ & & \\
\hline Depression & 11 & 50 & 11 & 50 & $\#<0.005$ \\
\hline schizophrenia & 6 & 40 & 9 & 60 & NS \\
\hline Delusional disorder & 0 & 0 & 5 & 100 & NS \\
\hline Personality disorder & 1 & 20 & 4 & 80 & NS \\
\hline Adjustment disorder & 5 & 100 & 0 & 0 & $€ \mathrm{p} \leq 0.001$ \\
\hline
\end{tabular}
\#chi square test
$€$ fisher's exact test
NS- Non significant

\section{DISCUSSION}

Incidence of DSH in patients with alcohol dependence syndrome was $25 \%$. This was similar to other recent Indian studies. ${ }^{[7,8,9]}$ DSH was found to be more in males in this study. Most patients with DSH belonged to younger age group with slight male preponderance in other Indian studies. ${ }^{[8,9]}$ But association between gender and deliberate self harm could not be statistically examined because of poor representation of 
females in this sample. Majority of the sample was less educated. This was also reported in other Indian studies. ${ }^{[2,7]}$

Most common method of DSH was poisoning (76\%) in our study. 68\% of alcohol dependent patients used organo phosphorus compounds for self harm. This is similar to other studies. ${ }^{[7,8,10]}$ More patients belonged to rural and agrarian background with farmers and agricultural laborers being the highest among males and housewives among females. Higher economic instability, risks associated with agriculture and easy availability and easy accessibility of pesticides explain these findings. Pesticide self-poisoning is now considered by the WHO to be the commonest method of fatal self-harm worldwide but it is rarely seen in the west. ${ }^{[2,6]}$ Restricted access to lethal means was associated with decline in suicide and in many cases also with overall suicide mortality. ${ }^{[11]}$

In this study $84 \%$ of alcohol dependent patients were intoxicated at the time of deliberate self harm replicating other studies. ${ }^{[8,9,10,12]}$ Ingestion of alcohol during or before the act sometimes can be considered to be part of the actual method of attempted suicide (when used to bring about unconsciousness, or to increase risk of a fatal outcome), as part of the preparation (to lower the threshold for engaging in an attempt because of disinhibition). ${ }^{[13]}$ In addition to increasing the likelihood of an attempt, alcohol may add considerably to the danger of overdoses. For those at high risk of repeated suicide attempts, previous self-harm involving alcohol may represent a warning sign and access to medication should be limited to prevent recidivism. ${ }^{[14]}$ In a qualitative interview of patients' experiences, several participants identified abstaining from alcohol as key to the resolution of deliberate self harm. Given the correlation between alcohol dependence and the risk of deliberate self harm, as well as the potential for brief interventions in emergency departments, this may be an issue for further research, particularly as some participants saw deliberate self harm as a way of accessing services. ${ }^{[15]}$

Majority of our patients had history of stressful situation immediately before the attempt. The negative life events, another distal risk factor was found to be statistically significant in association to deliberate self harm in alcohol dependent patients in this study. Association of masculinity and drinking and use of alcohol as a means of coping with stress by men are key factors behind the rising toll of alcohol-related premature mortality. ${ }^{[16]}$

$48 \%$ reported self harm as an impulsive act. This is similar to another study where the suicide attempters differed from non-attempters by higher levels of impulsive aggression and psychiatric co morbidity, particularly personality and depressive disorders. ${ }^{[7]}$

Past history of deliberate self harm was significantly associated with recurrent suicidal attempts in this study similar to observations by Mendelson. ${ }^{[17]}$ This risk increases with co morbid psychopathology and its severity. Our findings also show a statistically significant risk of repetition of DSH with co morbid psychiatric disorder. Persistence of intention to die among high number of DSH patients with psychiatric co morbidity has important bearing on the plan of management of these patients. ${ }^{[18]}$

Compared with non attempters, alcohol dependent attempters had history of deliberate self harm in a close relative. This was similar to other studies. ${ }^{[19}$, 20]

In our study $39 \%$ of alcohol dependent subjects with history of DSH had psychiatric co morbidity on assessment and this association was statistically significant. This was similar to other studies. [20, 21] Higher number of alcohol dependent patients with co morbid depressive disorders had attempted DSH in past 1 year when compared with patients with other co morbid psychiatric diagnosis. Alcohol dependent patients with co morbid depression may be at greater risk of psychosocial and interpersonal problems and attempted and completed suicide. ${ }^{[4]}$ A review 
reported that nearly one third of patients with major depressive disorder also had substance use disorders, and co morbidity yielded higher risk of suicide, greater social and personal impairment and other psychiatric conditions. ${ }^{[22]}$ Recognition and treatment of depression, especially in men, in primary care remains important in the prevention of suicidal behavior, as is the greater challenge of public education campaigns to improve public (and professional) understanding of mental illness and the effective treatments available.

In one study, alcohol dependent patients with independent major depression were found to be more likely to attempt suicide than those with substance-induced depression. ${ }^{[21]}$ However, major depression that occurs before the onset of a substance- related disorder might relate differently to suicidal behavior than major depression that occurs during the period of sustained abstinence. The latter is more likely to occur while patients are in treatment. ${ }^{[22]}$ This differentiation was not possible with our study and this warrants a future prospective to ponder.

All alcohol dependent patients with adjustment disorders resorted to deliberate self harm. Majority of them had psychosocial stressors leading to psychological distress, which in turn may act as a proximal risk factor for suicidal behavior. Proximal risk factors associated with acute intoxication are consistent with Baumeister's escape theory of suicide. ${ }^{[23]}$

A high rate of adjustment disorders in this study is probably a reflection of influence of socio cultural milieu. Similar rates were reported by another recent Indian study where adjustment disorder among males and depression and adjustment disorder among females were major psychiatric disorders. ${ }^{[7]}$

In our study, those alcohol dependent patients who attempted DSH had multiple psychiatric diagnoses (e.g., depression, Dissocial personality disorder) similar to Hesselbrock et al's observations. ${ }^{[24]}$ This indicates severity of illness. Presence of psychopathology elevates probability of self injurious behaviors and thoughts, and self- injury is associated with more symptoms and greater severity of psychopathology among both men and women. Alcohol dependence, depression and antisocial personality disorder form the formidable triad towards self-destruction. Having a personality disorder may be determinant of suicidal behavior in several ways by predisposing to major psychiatric disorders like depressive disorder or alcohol dependence by leading to difficulties in relationship and social adjustment. [25]

But only 5 among 25 alcohol dependent patients who committed deliberate self harm had bipolar affective disorder. This may be because suicidal attempt is rare in manic phase.

Universal preventive interventions are directed toward the entire population; selective interventions are directed toward individuals who are at greater risk for suicidal behavior; and indicated preventions are targeted at individuals who have already begun self-destructive behavior. ${ }^{[11]}$ This study is a beginning in this venture which provides an understanding of the burden of DSH from alcohol addiction, emphasizes the need for early identification and intervention, and the need for training primary health care service providers to effectively assess and manage alcohol dependence in primary health care settings.

4.1. Limitation: This was a cross sectional study from a hospital set up which limits the generalizability of the observations. Further current study did not use any structured instruments for assessing patients. Lifetime comorbidity associations and retrospective evaluation of DSH especially when self reported may be subject to recall bias. Tendency of patients and families to keep problems hidden could lead to underestimation of results. A large scale community based study in the same area with validated instruments and questionnaires will give an actual picture of the problem in this community. 
4.2. Strength of the study: Cross sectional evaluation of 100 patients with alcohol dependence was done. This was a decent number for any time limited cross sectional research. This study was able to highlight the incidence and pattern of deliberate self harm in alcohol dependent patients.

\section{CONCLUSION}

All patients presenting after deliberate self-harm need to be carefully screened for alcohol disorders and for co morbid psychiatric diagnoses. This study suggests that depressive disorder and alcohol dependence are critical combinations for high risk of deliberate self harm. Treatment of deliberate self-harm patients with alcohol disorders should include the treatment of any co morbid psychiatric illness especially depressive disorder. Effective strategies of stress management may help in the reduction of prevalence of psychiatric co morbidities in alcohol dependence and their culmination in deliberate self harm. In addition to increasing the likelihood of an attempt, alcohol may add considerably to the danger of organo phosphorus poisoning and hence appropriate legislative measures should be implemented to restrict its availability. Abstaining from alcohol may be considered as a key to the resolution of deliberate self harm.

Conflict of interest: None declared.

Ethical approval: The study was approved by the institutional Ethics committee.

\section{REFERENCES}

1. Hawton K, Catalan J. Attempted Suicide: A Practical Guide to its Nature and Management. Oxford: Oxford University Press; 1987.

2. Chowdhary AN, Banarjee S, Brahma A. Pesticide poisoning in non fatal deliberate self-harm - a public health issue. Indian Journal of Psychiatry 2007;49:117-20.
3. Hufford MR. Alcohol and suicidal behavior. Clinical Psychology Review 2001;21:797-811.

4. Haw C, Houston K, Townsend E, Hawton K. Deliberate self-harm patients with alcohol disorders: characteristics, treatment, and outcome. Crisis 2001;22:93-101.

5. Powell KE, Kresnow MJ, Mercy JA et al. Alcohol consumption and nearly lethal suicide attempts. Suicide and Life Threat Behavior 2001; 32: 30-41.

6. Venkoba RA. Suicidology: The Indian context. In: Aggarwal SP, Goel DS, Ichhpujani RL, Salhan RN, Shrivastava S, editors. Mental health: An Indian perspective, 1946-2003. New Delhi: Directorate General of Health Services/Ministry of Health and Family Welfare; 2004. p. 279-83.

7. Patil SS, Yadavannavar MC, Chaukimath SP. Deliberate self harm and psychiatric co morbidity: a hospital based study. Asian Journal of Experimental Biological Science 2011;2:367-71.

8. Koganti CT, Mittal S, Safeekh A T, Rao $\mathrm{P}$, Chandini. Prevalence of Deliberate Self-Harm in Alcohol Dependence Syndrome Patients. International Journal of Scientific and Research Publications 2015;5:1-6.

9. Davis Manuel, Linus Francis, K. S. Shaji .Prevalence and risk factors for psychiatric co-morbidity in patients with alcohol dependence syndrome. Journal of Evidence based Medicine and Healthcare 2015;36: 5704-11.

10. Srivatsava MK, Sahoo RN, Ghotkar LH et al. Risk factors associated with attempted suicide: a case control study. Indian Journal of Psychiatry 2004;46:33-8.

11. Nordentoft M. Prevention of suicide and attempted suicide in Denmark. Epidemiological studies of suicide and intervention studies in selected risk 
groups. Danish Medical

Bulletin.2007;54:306-69.

12. Cheryl J, Cherpitel, Guilherme LG et al. Acute alcohol use and suicidal behavior: a review of the literature. Alcoholism Clinical \& Experimental Research 2004;28:18-28.

13. Haw C, Hawton K, Casey D et al. Alcohol dependence, excessive drinking and deliberate self-harm: trends and patterns in Oxford, 1989-2002. Epub 2005; 40: 96471.

14. Marc A. Schuckit M.D. Alcohol-Related Disorders. In Comprehensive Text Book of Psychiatry. Edition 9. Edited by. Sadock BJ, Sadock VA. Lippincott Williams and Wilkins. New York. 2009: 1269-88.

15. Modesto-Lowe V, Brooks D, Ghani M. Alcohol dependence and suicidal behavior: from research to clinical challenges. Harvard Review of Psychiatr.2006; 14: 241-8.

16. Vikram Patel. Alcohol Use and Mental Health in Developing Countries. Annual of Epidemiology 2007;17: 87-92.

17. Mendelson EF. Alcohol-related psychiatric emergencies: their characteristics and care at a walk-in clinic. British Journal of Psychiatry 198;150:121-4.

18. Schuckit MA. Primary men alcoholics with histories of suicide attempts. Journal of Study of Alcoholism. 1986 47:78-81.

19. Roy A. Relation of family history of suicide to suicide attempts in alcoholics. American Journal of Psychiatry 2000; 157:2050-1.

20. Khisty N, Raju MSV, Tampi UR. A study of co morbid psychiatric disorder in alcoholism. Indian Journal of Psychiatry $2001 ; 43$.
21. Chaudhary B, Das P. Depression in alcoholics - relationship with socio demographic variables and abstinence. Indian Journal of Psychiatry 2001;43.

22. Davis L, Uezato A, Newell JM et al. Major depression and co morbid substance use disorders. Current Opinion in Psychiatry 2008; 21:14-8.

23. Baumeister RF. Suicide as escape from self. Psychol Rev 1990;97:90-113.

24. Hesselbrock M, Hesselbrock V, Syzmanski K. Suicide attempts and alcoholism. Journal of Study of Alcoholism 1988;49:436-42.

25. Pirkola SP, Suominen K, Isometsa ET. Suicide in alcohol-dependent individuals: epidemiology and management CNS Drugs. 2004;18:423-36 